\title{
Direct imaging of Earth-like planets: why we care about exozodis
}

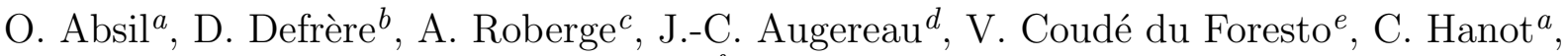 \\ C. Stark ${ }^{f}$ and J. Surdej ${ }^{a}$ \\ ${ }^{a}$ IAGL, Université de Liège, 17 Allée du Six Août, B-4000 Liège, Belgium; \\ ${ }^{b}$ Max-Planck-Institut für Radioastronomie, Auf dem Hügel 69, 53121 Bonn, Germany; \\ ${ }^{c}$ Exoplanets and Stellar Astrophysics Laboratory, NASA Goddard Space Flight Center, \\ Code 667, Greenbelt, MD 20771, USA; \\ ${ }^{d}$ LAOG-UMR 5571, CNRS and Université Joseph Fourier, BP 53, F-38041 Grenoble, France; \\ ${ }^{e}$ LESIA-UMR 8109, CNRS and Observatoire de Paris-Meudon, 5 place J. Janssen, F-92195 \\ Meudon, France; \\ ${ }^{f}$ Department of Physics, University of Maryland, Box 197, 082 Regents Drive, College Park, \\ MD 20742-4111, USA
}

\begin{abstract}
The presence of large amounts of exozodiacal dust around nearby main sequence stars is considered as a potential threat for the direct detection of Earth-like exoplanets (exoEarths) with future space-based coronagraphic and interferometric missions. In this paper, we estimate the amount of exozodiacal light that can be tolerated around various stellar types without jeopardizing the detection of exoEarths with a space-based visible coronagraph or a free-flying mid-infrared interferometer. We also address the possible effects of resonant structures in exozodiacal disks. We then review the sensitivity of current ground-based interferometric instruments to exozodiacal disks, based on classical visibility measurements and on the nulling technique. We show that the current instrumental performances are not sufficient to help prepare future exoEarth imaging missions, and discuss how new groundor space-based instruments could improve the current sensitivity to exozodiacal disks down to a suitable level.
\end{abstract}

Keywords: Circumstellar matter, planetary systems, high angular resolution

\section{INTRODUCTION}

As recognized by the European and American Space Agencies, exozodiacal dust in the habitable zone around nearby stars is a source of background flux that must be considered in any effort to image terrestrial planets. The strength of the exozodiacal light intensity in typical stellar systems is not known. In the Solar System, zodiacal dust is produced by collisions among asteroids and by outgassing of comets. The zodiacal dust cloud is in fact the most luminous component after the Sun, and interestingly, the signature of the Earth is clearly seen as clumps in the zodiacal dust disk. ${ }^{1}$ These facts reveal two faces of exozodiacal dust disks. On the one hand, exozodiacal dust might prevent the detection of exoEarths with future space-based facilities. On the other hand, structures in exozodiacal clouds might be signposts of unseen perturbing planets and small body populations that produce visible dust.

Exozodiacal clouds are related to debris disks, clouds of rocks and cold dust orbiting at a few tens of AU from main sequence stars. A significant fraction $(\sim 15 \%)$ of nearby solar-type stars are already known to host such cold debris disks, which are thought to be aftermaths of planet formation and to derive from collisions in extrasolar Kuiper belts. A fleet of space facilities (HST, ISO, Spitzer and now Herschel) has been revolutionizing the study of debris disks. Many structures and asymmetries in spatially resolved debris disks have been used to infer the presence of yet unseen planets in these systems. The recent detections by Kalas et al. ${ }^{2}$ and Lagrange et al. ${ }^{3}$ of giant planets respectively orbiting the bright stars Fomalhaut (at the inner edge of the dust ring) and

Send correspondence to O.A. (absil@astro.ulg.ac.be) 
beta Pic (in the inner part of the debris disk) come as a confirmation of the theoretical approaches developed by several teams over the last decade, in a sense reproducing the experience by Galle in 1846 who captured the first image of Neptune based on the calculations by Adams and Le Verrier.

Unfortunately, nearly nothing is known about exozodiacal clouds around nearby stars, namely on dust in the inner planetary region of the extrasolar systems that should stem from "exoasteroids" and "exocomets". This ignorance could significantly affect the development and design of future exoEarth imaging missions. The present paper discusses the impact of exozodiacal disks on the performance of these missions, and brings up some possible ways to solve the "exozodi problem".

\section{IMPACT OF EXOZODIS ON EXOEARTH IMAGING WITH OPTICAL TELESCOPES}

The most obvious effect of exozodiacal dust at optical wavelengths on direct observations of Earth-like planets is to increase the overall shot noise and hence the required exposure times. Light scattered off the local zodiacal dust and the exozodiacal dust around nearby stars will be blended with the planet signal in both images and spectra. The wider the final PSF, the more background will be mixed in. This background will likely be the largest source of noise, assuming the stellar light is canceled by $10^{-10}$ or more at the planet pixel. The magnitude of the exozodi emission within the point-spread function of a 4-meter optical telescope is $V \approx 28$, assuming a Solar System-twin at $10 \mathrm{pc}$ viewed at $60^{\circ}$ inclination. This is about two magnitudes brighter than the Earth viewed at quadrature.

In the background limited case, ${ }^{4}$ the exposure time to image a planet is

$$
t=\frac{2 n_{x} \lambda^{2}}{\pi F_{0} \Delta \lambda D^{4} T}\left(\frac{S}{N}\right)^{2} 10^{0.8\left(M_{p}+5 \log d-5\right)}\left[\left(\frac{206265^{\prime \prime}}{1 \mathrm{rad}}\right)^{2}\left(10^{-0.4 z}+\epsilon 10^{-0.4 x}\right)+\zeta 10^{\left.-0.4 m_{\star} \mathrm{PSF}_{\mathrm{peak}}\right]}\right.
$$

where $n_{x} \equiv 1 /$ "sharpness" = number of pixels in a critically sampled diffraction-limited image, $\lambda=$ central wavelength of the image bandpass, $F_{0}=$ specific flux for zero magnitude in the image bandpass, $\Delta \lambda=$ bandpass width, $D=$ diameter of the telescope aperture in meters, $T=$ total facility throughput, $S / N=$ signal-to-noise, $M_{p}=$ absolute magnitude of the planet, $d=$ distance to the system in pc, $z=$ surface brightness of the zodiacal dust in mag $\operatorname{arcsec}^{-2}, \epsilon=$ exozodi brightness in units of the surface brightness of a Solar System-twin exozodi disk, $x=$ surface brightness of a Solar System-twin exozodi disk in mag $\operatorname{arcsec}^{-2}, \zeta=$ contrast level in the detection zone with respect to the theoretical Airy peak of the stellar image, $m_{\star}=$ stellar apparent magnitude, $\mathrm{PSF}_{\text {peak }}=\frac{\pi D^{2}}{4 \lambda^{2}}=$ theoretical peak brightness of the stellar point-spread function. In this equation, the first term in the square brackets accounts for noise from the local zodiacal background, the second from the exozodi background, and the third from the residual unsuppressed stellar light. Other noise sources (e.g. read and dark noise) are assumed to be negligible.

Equation 1 shows that the exposure time is linearly proportional to the exozodi surface brightness $(\epsilon)$. Obviously, if the exozodi is too bright, the integration time to get the required planet SNR becomes prohibitively long. How do various optical mission concepts perform under varying levels of exozodi? Using publicly available information, it is very hard to come up with estimates that can be reasonably compared. In addition, most groups assume a fixed exozodi level for calculating their mission performance, typically the level of a Solar System-twin exozodi disk, which is not tremendously informative. There is a strong need for uniform mission performance analysis that pushes to high exozodi levels.

That being written, Figure 1 illustrates the decline in mission performance with increasing exozodi brightness at optical wavelengths. These preliminary calculations were done assuming the parameters of the New Worlds Observer mission concept (4-m telescope, broad-band imaging channel covering 500 to $700 \mathrm{~nm}$ ), but the general behavior should be similar for other optical wavelength missions using either external occulters or internal coronagraphs. However, it is important to note that these calculations include only statistical errors due to local zodiacal background, exozodiacal background, and unsuppressed starlight. Possible systematic errors associated with modeling light scattered off a non-uniform exozodiacal dust distribution and removing it from a planet image have not been thoroughly characterized for optical missions. Furthermore, confusion between planets and exozodiacal dust structures like resonant clumps remains poorly studied, although initial attempts to evaluate its impact on mission performance have been carried out. ${ }^{5}$ 


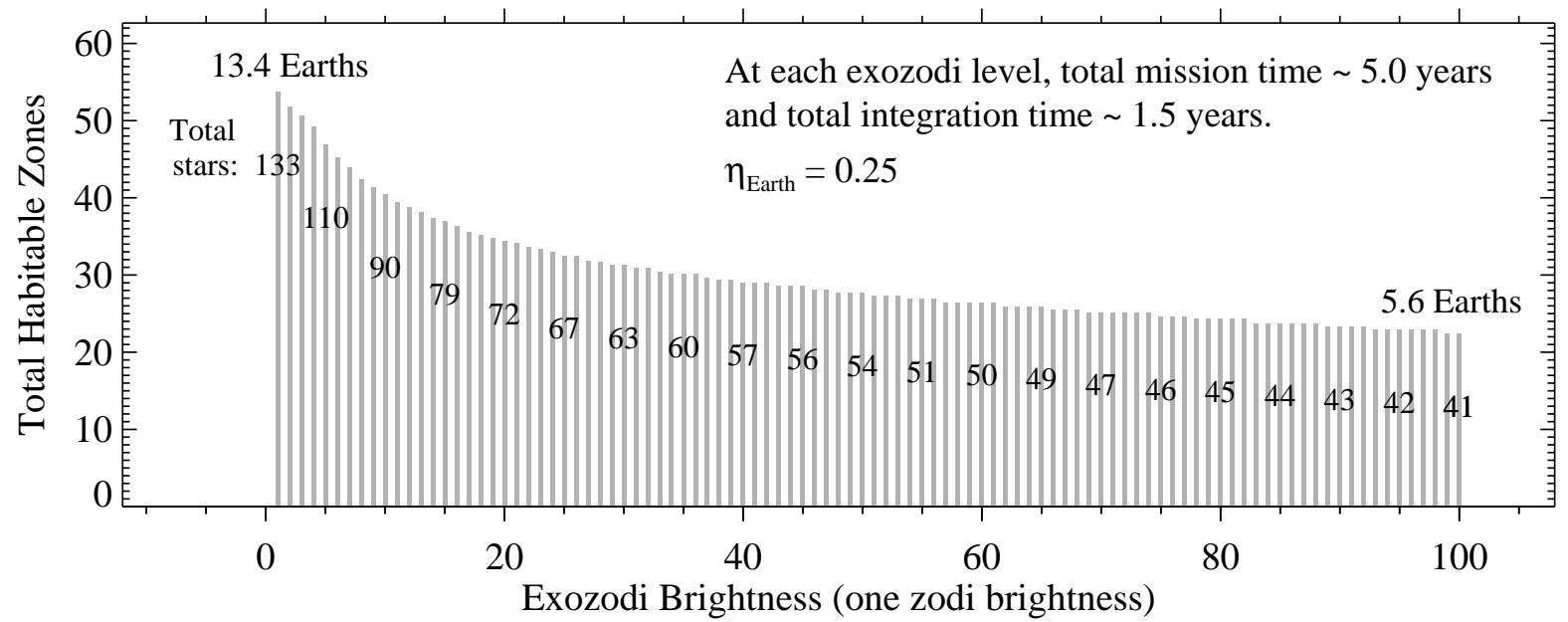

Figure 1. Total number of habitable zones searched vs. exozodi surface brightness for actual stars within 15 pc of the Sun. Each grey bar represents a possible observing program, assuming $\eta_{\oplus}=0.25$ (fraction of stars with Earth-like planets) and the parameters of the New Worlds Observer mission concept (for more details see Turnbull et al., in preparation). The numbers superimposed on some of the grey bars are the total numbers of stars observed in the programs. The $\mathrm{y}$-axis represents the total number of habitable zones searched, which is the sum of the completeness values for all the individual stars observed. A star's completeness value is the probability that a habitable zone planet would be detected in a single visit, given the possible range of system inclinations and planet eccentricities. The expected number of exoEarths characterized is the total number of habitable zones searched times $\eta_{\oplus}$. To generate a program for each exozodi level, stars were chosen in order of decreasing weighting factor (completeness / exposure time) until the total on-target integration time reached 1.5 years or the total mission time reached 5 years, whichever came sooner. The total integration times were calculated assuming that for $\eta_{\oplus} \times 100=25 \%$ of the targets, a spectrum with $S / N \geq 10$ and $R=100$ was obtained in addition to the imaging observation. Total mission times include 11 days per target for moving the external occulter.

\section{IMPACT OF EXOZODIS ON EXOEARTH IMAGING WITH MID-INFRARED INTERFEROMETRY}

The mid-infrared wavelength range presents several advantages for Earth-like planet characterization. In addition to including spectral bands of water, carbon dioxide and ozone, the contrast between a star and an exoEarth is only $\sim 10^{7}$ whereas it is $\sim 10^{10}$ in the visible. However, resolving the habitable zone around nearby stars in the mid-infrared would require a very large telescope with a diameter of at least $20 \mathrm{~m}$. Space-based interferometry is therefore considered as the most promising technique to achieve this goal. A large effort has been carried out during the past decade to define a design that provides excellent scientific performance while minimizing cost and technical risk. This has resulted in a convergence and consensus on a single mission architecture consisting of a non-coplanar X-array, called Emma, using four collector spacecraft and a single beam combiner spacecraft. ${ }^{6}$ Such a design enables the implementation of phase chopping, a technique which suppresses from the final output all sources having point-symmetric brightness distributions.

The impact of exozodis on the mission performance is then twofold: on one hand, their point-symmetric component contributes to the overall shot noise and can therefore drive the required integration time to detect exoEarths, while on the other hand, asymmetric structures in exozodis (such as resonant clumps) are not suppressed by phase chopping and thereby contribute as possible biases (or false positives), which could prevent the detection of small planets.

Considering point-symmetric exozodiacal disks, the number of nearby main sequence stars that can be surveyed for Earth-like planets decreases with respect to the dust density, as shown in Figure 2 for an Emma X-array interferometer with different aperture sizes. In order to preserve $75 \%$ of the mission outcome, i.e., surveying the habitable zone of at least 150 targets with $>90 \%$ completeness during the 2 -yr search program, point-symmetric exozodis of about 100 zodis can be tolerated in the nominal configuration (2-m aperture telescopes). For larger 


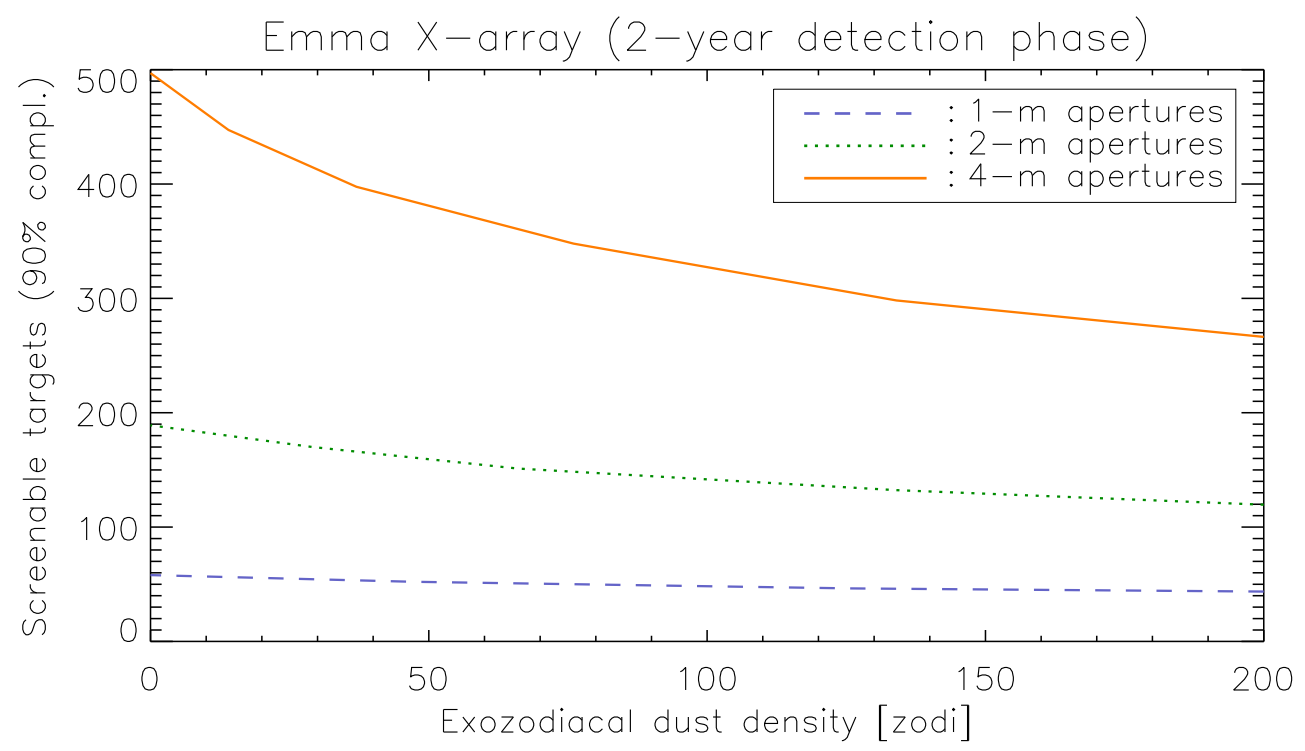

Figure 2. Number of nearby main sequence stars that can be surveyed for Earth-like planets as a function of the exozodiacal dust density, assuming a space-based nulling interferometer in the Emma X-array configuration and various telescope diameters. Integration times have been computed assuming a broadband detection $(S / N>5)$ and overhead losses of $70 \%$. Total mission times also include 6 hours for re-targeting the array and a specific time allocation for each spectral type $(10 \% \mathrm{~F}, 50 \% \mathrm{G}, 30 \% \mathrm{~K}$ and $10 \% \mathrm{M})$.
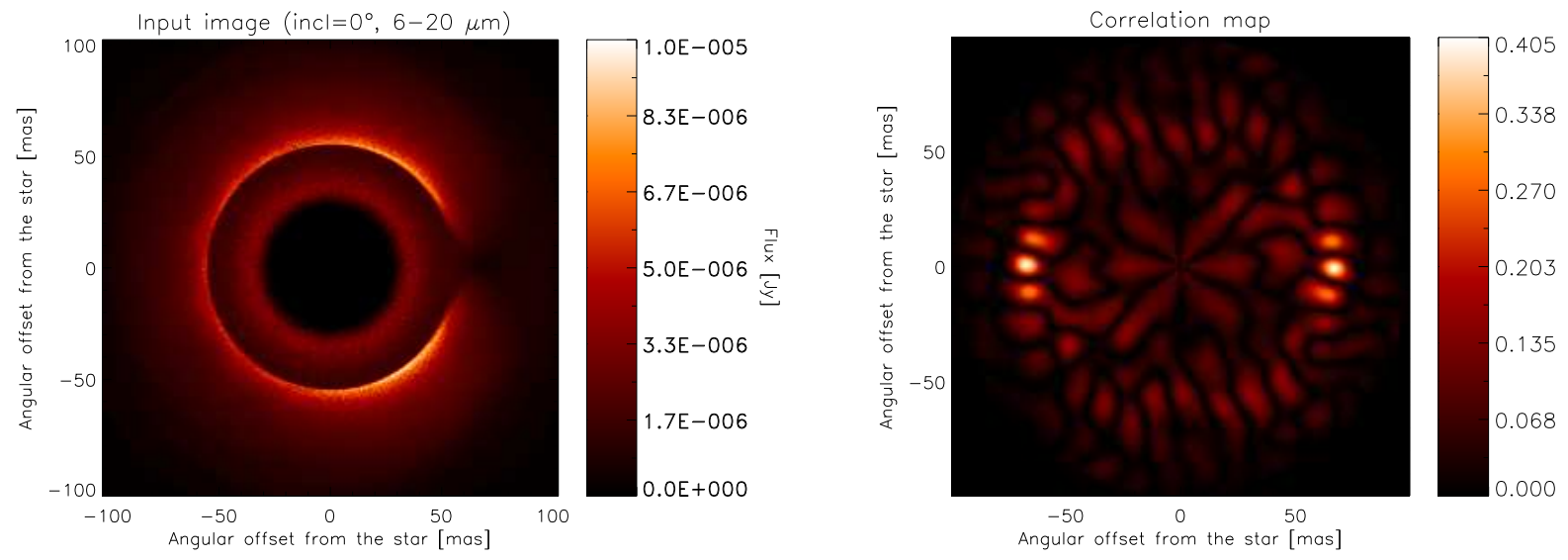

Figure 3. Simulated image of the thermal flux produced by a 10-zodi face-on exozodiacal dust cloud in the 6 - $20 \mu \mathrm{m}$ range, around a G2V star located at 15 pc. The simulation includes the presence of an Earth-mass planet located at 1 AU on the $\mathrm{x}$-axis, and assumes a Dohnanyi size distribution for the dust grains. ${ }^{7}$ Right: Corresponding dirty map computed for an Emma X-array nulling interferometer, obtained by cross-correlating the measured signal with templates for the expected signal from a point source at each location on the sky plane. ${ }^{8}$ 
aperture telescopes (e.g., 4-m diameter), the relative impact of the exozodiacal dust density on the mission performance is larger and only 60 zodis can be tolerated in order to preserve $75 \%$ of the original mission outcome (which represents about 500 stars searched in this case).

The possible presence of asymmetric structures in exozodiacal disks (e.g., clumps or offset) is more problematic. In order to assess their influence on the detectability of exoEarths, we have used synthetic images of exozodiacal disks including the resonant structures created by an exoEarth orbiting at 1 AU around a Sun-like $\operatorname{star}^{7}$ (Figure 3, left). Most notable among the asymmetric structures is the "hole" surrounding the planet position. The hole in the dust distribution contributes significantly to the final detected signal after phase chopping. It actually appears at similar frequencies as the planet in the rotation-modulated signal, and can therefore produce a significant bias (or false-positive) in the planet detection process (see dirty map in the right part of Figure 3).

We adopt a standard criterion for planet detection, using for the noise level the RMS deviation of the pixel counts in the dirty map within an annulus of width equal to the PSF size at half maximum. We require the planetary signal to be at least 5 times larger than this noise level. The tolerable dust density then goes down to about 15 times the solar zodiacal dust density for face-on systems in order to detect an Earth-like planet. The tolerable exozodi density is even lower for large disk inclinations, down to a few zodis. ${ }^{8}$ This upper limit on the tolerable exozodiacal dust density gives an estimation of the typical sensitivity that precursor instruments will need to reach on exozodiacal disks in order to prepare the scientific program of future exoEarth characterization missions (i.e., typically 10 zodis).

\section{CURRENT EXOZODIACAL DISK DETECTION EFFORTS}

Two main techniques are being used to evaluate the amount of exozodiacal dust around main sequence stars: infrared spectro-photometry and infrared interferometry.

\subsection{Infrared spectro-photometry}

Infrared spectro-photometry can reveal the presence of an excess emission on top of the expected stellar photospheric flux. This requires high-accuracy photometry in the mid-infrared regime, which is generally done from space, e.g., using the various instruments on board the Spitzer Space Telescope. In particular, the Multiband Imaging Photometer for Spitzer (MIPS) at $24 \mu \mathrm{m}$ and the Infrared Spectrometer (IRS) between 5 and $34 \mu \mathrm{m}$ have been used to search for the signature of warm $(\geq 200 \mathrm{~K})$ dust around nearby main sequence stars. In both cases, the intrinsic accuracy of Spitzer spectro-photometry limits the sensitivity to exozodiacal disks about 1000 times more luminous than the solar zodiacal cloud, assuming the same brightness distribution. ${ }^{9,10}$ It must be noted that, even with an infinite accuracy on the measured photometry, this technique would still be limited by the capability to predict the mid-infrared stellar photospheric flux, so that exozodiacal disks fainter than a few hundreds of zodis are not within reach.

\subsection{Infrared interferometry}

The second way to detect exozodiacal disks overcomes this limitation by angularly separating the signal of the star from that of its surrounding dust disk. The requested angular resolution can generally be achieved only with infrared interferometry, as exozodis are generally separated by less than 100 mas from their parent stars (except for very nearby stars, with $d<10 \mathrm{pc}$ ). Two main types of interferometers are contributing to exozodi surveys: high-accuracy near-infrared interferometers and mid-infrared nulling interferometers.

High accuracy near-infrared interferometers such as CHARA/FLUOR, VLTI/VINCI or IOTA/IONIC have been used to search for small visibility drops at short baselines around unresolved main sequence stars, which would be the sign of resolved circumstellar emission. Successful attempts have been reported in a few cases already. ${ }^{11-15}$ The detected K-band excesses are interpreted as the signature of hot circumstellar dust, and the equivalent densities are generally of a few thousands of zodis. The current accuracy of this technique, mainly limited by calibration issues, is around 1000 zodis. Although there is still room for improvement, it is not expected that accuracies below 100 zodis could be reached. 


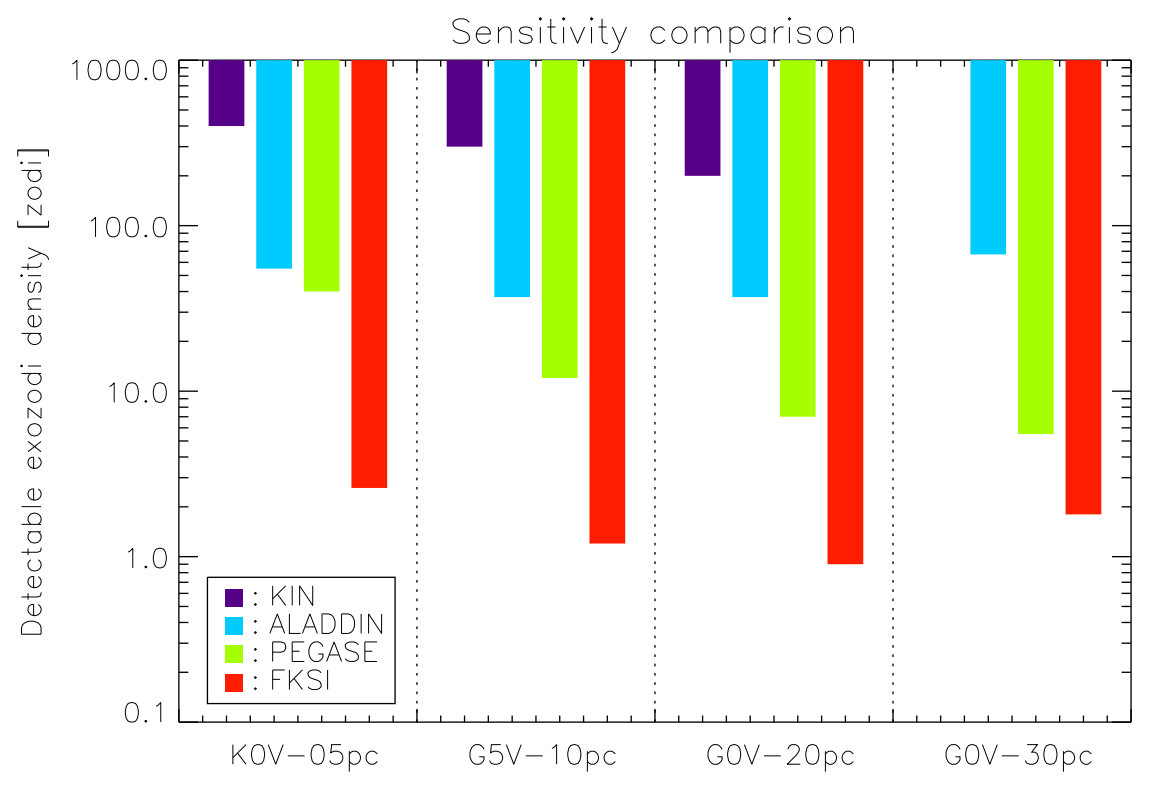

Figure 4. Measured (KIN) or simulated (ALADDIN, Pegase, FKSI) sensitivity of various ground- and space-based nulling interferometers, in terms of the smallest exozodiacal dust density that can be detected at $3 \sigma$ around various nearby solar-type stars.

In the case of mid-infrared interferometry, the nulling technique has been implemented with BLINC on the $\mathrm{MMT}^{16}$ and on the Keck Interferometer Nuller. ${ }^{17}$ In both cases, a typical accuracy of $10^{-3}$ on the disk/star contrast can be reached, leading to a sensitivity of about 300 zodis. ${ }^{18}$ These observations are however still restricted to a small number of targets, due either to limited observing time or to the limiting magnitude of the instrument. Improvements of the nulling technique are foreseen in the near- to mid-term future with projects such as the LBTI at Mount Graham (Arizona) ${ }^{19}$ or the ALADDIN instrument considered for Dome C (Antarctica). ${ }^{20}$ Both nulling instruments are expected to reach sensitivities down to about 30-50 zodis, respectively in the $\mathrm{N}$ band and the L band. Space-based instruments are also being considered, such as the Pegase ${ }^{21}$ and $\mathrm{FKSI}^{22}$ projects. Thanks to a much lower background level and to the absence of atmospheric turbulence, these instruments should reach a sensitivity down to about 1 zodi for nearby Sun-like stars. ${ }^{23}$ The expected sensitivities of these various instruments are summarized in Figure 4.

\section{CURRENT EXOZODIACAL DISK MODELING EFFORTS}

The current exozodi modeling efforts follow two main paths. On the one hand, radiative transfer codes for optically thin disks are being employed to reproduce the scarce exozodi measurements, in particular near- and mid-infrared interferometric data (see e.g. Figure 5), and show that the solar system zodiacal spectrum does not match the spectral energy distributions (SEDs) of detected exozodiacal disks. The fit to the $2.2 \mu \mathrm{m}$ excesses with the solar system zodiacal model indeed indicates densities a few thousand times larger than the zodiacal density (e.g., about 3000 zodis for Vega and 5000 zodis for Fomalhaut), but this model predicts a much too large flux (by about an order of magnitude) in the mid-infrared compared to the observations. Dust much closer to the star (down to the sublimation radius at a fraction of an AU), and with a sufficient amount of refractory material, are required to shift the spectrum to shorter wavelengths and match the current data sets. Spectral decomposition techniques are also being developed to reproduce extreme Spitzer/IRS spectra showing unusually large amounts of warm dust and clear spectral solid-state features. ${ }^{24}$

On the other hand, dynamical models employing classical N-body codes are being used to assess the influence of planets on the shape of exozodiacal disks and discuss the dust production mechanisms. Some models 


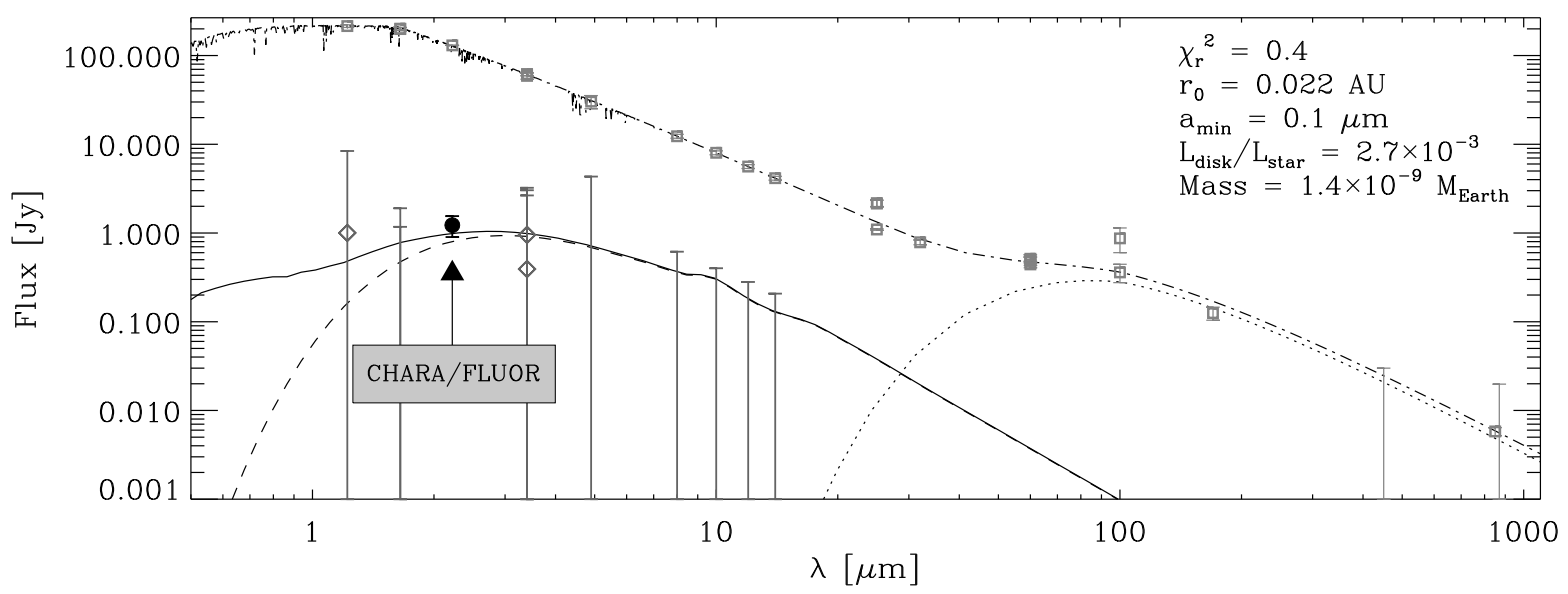

Figure 5. A possible fit of the photometric excesses (represented with diamonds) and interferometric observations (filled circle at $2.2 \mu \mathrm{m}$ ) of the $\tau$ Ceti exozodi. ${ }^{12}$ Dashed line: thermal emission from the best-fit disk model. Solid line: includes the scattered light contribution. Dotted line: approximative blackbody fit to the long-wavelength excesses produced by a Kuiper-like belt. Dashed-dotted line: total emission from the star-disk system.

for instance simulate the sculpting of an asteroid/dust belt by a planet due to their capture in mean motion resonances. ${ }^{7}$ Larger scale models, involving the outward migration of a planet toward a Kuiper belt are currently being developed, basically relying on the assumption that exozodis are fed by the outer, much more massive disk regions, thereby linking the inner and outer regions of planetary systems. This can either be due to a sudden event, e.g., a Nice LHB-like model, ${ }^{25}$ or be more progressive (Vandeportal et al., in prep.). The amount of dust produced can be assessed for individual systems following this methodology, but it is very sensitive to the assumed planetary system architecture. Finally, collisional models, using statistical approaches, can evaluate the lifetime of asteroid belts due to collisions. ${ }^{26,27}$ An ISSI working group has been assembled to further discuss the modeling of exozodis (see http://www.issibern.ch/teams/exodust/).

\section{CONCLUSIONS}

This short review illustrates how the final SNR for direct exoEarth detection depends on the quantity of exozodiacal dust around main sequence stars. On one hand, it drives the required integration time to detect the planetary signal as soon as its density reaches a few tens of zodis, and on the other hand, its potential asymmetries induce biases and false positives, which in turn demand the planetary systems to be observed longer in order to extract the actual planetary signal. If space missions had an unlimited lifetime, this wouldn't be a major issue, as one would just skip the inappropriate targets, or integrate longer to eventually reveal their planets. However, space missions are limited in time, and the exozodi issue could thus become a major hurdle in case bright exozodis are common. The sensitivity of current exozodi finders ( 300 zodis at best $)$ is not appropriate to assess whether exozodis in the 10-100 zodi range are common or not. A dedicated effort to solve this question is therefore mandatory.

Three possible avenues have been identified to make sure that exoEarth imaging missions will be capable of reaching their goals:

- Perform an exozodi survey with a sensitivity of $\sim 30$ zodis on a statistically meaningful sample of main sequence stars to constrain the distribution of exozodi brightness down to an appropriate level. Space missions can then be designed (in terms of aperture size and mission lifetime) to cope with the inferred mean exozodi level.

- Measure the exozodi level with an accuracy of $\sim 10$ zodis on all the candidate mission targets, once the targets have been identified (e.g., Sun-like stars hosting exoEarth(s), detected through high-precision astrometric or radial velocity surveys). 
- Use the fact that the statistical distribution of cold debris disks will soon be known at a level similar to the density of the solar Kuiper belt thanks to Herschel. Extrapolating the statistics of cold debris disks towards that of warm exozodis is however not straightforward based on theoretical models, and needs to be backed up by observational data anyway.

Defining an optimized strategy to solve the "exozodi problem" will be tackled in the coming months by the Debris Disks and Exozodis Study Analysis Group of the NASA Exoplanet Exploration Program (ExoPAG).

\section{ACKNOWLEDGMENTS}

O.A. acknowledges the financial support of F.R.S.-FNRS (Postdoctoral Researcher). O.A., D.D, C.H. and J.S. acknowledge support from the "Communauté Française de Belgique - Actions de Recherche Concertées Académie universitaire Wallonie-Europe". This research was partly funded by the International Space Science Institute ("Exozodiacal Dust Disks and Darwin" working group).

\section{REFERENCES}

[1] Kelsall, T., Weiland, J. L., Franz, B. A., Reach, W. T., Arendt, R. G., Dwek, E., Freudenreich, H. T., Hauser, M. G., Moseley, S. H., Odegard, N. P., Silverberg, R. F., and Wright, E. L., "The COBE Diffuse Infrared Background Experiment Search for the Cosmic Infrared Background. II. Model of the Interplanetary Dust Cloud," ApJ 508, 44-73 (Nov. 1998).

[2] Kalas, P., Graham, J. R., Chiang, E., Fitzgerald, M. P., Clampin, M., Kite, E. S., Stapelfeldt, K., Marois, C., and Krist, J., "Optical Images of an Exosolar Planet 25 Light-Years from Earth," Science 322, 1345(Nov. 2008).

[3] Lagrange, A., Gratadour, D., Chauvin, G., Fusco, T., Ehrenreich, D., Mouillet, D., Rousset, G., Rouan, D., Allard, F., Gendron, É., Charton, J., Mugnier, L., Rabou, P., Montri, J., and Lacombe, F., "A probable giant planet imaged in the $\beta$ Pictoris disk. VLT/NaCo deep L'-band imaging," A\&A 493, L21-L25 (Jan. 2009).

[4] Brown, R. A., "Single-Visit Photometric and Obscurational Completeness," ApJ 624, 1010-1024 (2005).

[5] Savransky, D., Kasdin, N. J., and Vanderbei, R. J., "An evaluation of the effects of non-uniform exozodiacal dust distributions on planetary observations," in [Techniques and Instrumentation for Detection of Exoplanets IV], Proc. SPIE 7440 (Aug. 2009).

[6] Cockell, C. S., Herbst, T., Léger, A., Absil, O., Beichman, C., Benz, W., Brack, A., Chazelas, B., Chelli, A., Cottin, H., Coudé du Foresto, V., Danchi, W., Defrère, D., den Herder, J., Eiroa, C., Fridlund, M., Henning, T., Johnston, K., Kaltenegger, L., Labadie, L., Lammer, H., Launhardt, R., Lawson, P., Lay, O. P., Liseau, R., Martin, S. R., Mawet, D., Mourard, D., Moutou, C., Mugnier, L., Paresce, F., Quirrenbach, A., Rabbia, Y., Rottgering, H. J. A., Rouan, D., Santos, N., Selsis, F., Serabyn, E., Westall, F., White, G., Ollivier, M., and Bordé, P., "Darwin: an experimental astronomy mission to search for extrasolar planets," Experimental Astronomy 23, 435-461 (Mar. 2009).

[7] Stark, C. C. and Kuchner, M. J., "The Detectability of Exo-Earths and Super-Earths Via Resonant Signatures in Exozodiacal Clouds," ApJ 686, 637-648 (Oct. 2008).

[8] Defrère, D., Absil, O., den Hartog, R., Hanot, C., and Stark, C., "Nulling interferometry: impact of exozodiacal clouds on the performance of future life-finding space missions," $A \mathscr{G} A$ 509, A9 (Jan. 2010).

[9] Bryden, G., Beichman, C. A., Trilling, D. E., Rieke, G. H., Holmes, E. K., Lawler, S. M., Stapelfeldt, K. R., Werner, M. W., Gautier, T. N., Blaylock, M., Gordon, K. D., Stansberry, J. A., and Su, K. Y. L., "Frequency of Debris Disks around Solar-Type Stars: First Results from a Spitzer MIPS Survey," ApJ 636, 1098-1113 (Jan. 2006).

[10] Lawler, S. M., Beichman, C. A., Bryden, G., Ciardi, D. R., Tanner, A. M., Su, K. Y. L., Stapelfeldt, K. R., Lisse, C. M., and Harker, D. E., "Explorations Beyond the Snow Line: Spitzer/IRS Spectra of Debris Disks Around Solar-type Stars," ApJ 705, 89-111 (Nov. 2009). 
[11] Absil, O., Di Folco, E., Mérand, A., Augereau, J.-C., Coudé du Foresto, V., Aufdenberg, J. A., Kervella, P., Ridgway, S. T., Berger, D. H., ten Brummelaar, T. A., Sturmann, J., Strumann, L., Turner, N. H., and McAlister, H. A., "Circumstellar material in the Vega inner system revealed by CHARA/FLUOR," $A \& A$ 452, 237-244 (2006).

[12] Di Folco, E., Absil, O., Augereau, J.-C., Mérand, A., Coudé du Foresto, V., Thévenin, F., Defrère, D., Kervella, P., ten Brummelaar, T. A., McAlister, H. A., Ridgway, S. T., Sturmann, J., Strumann, L., and Turner, N. H., "A near-infrared interferometric survey of debris-disk stars. I. Probing the hot dust content around $\epsilon$ Eri and $\tau$ Cet with CHARA/FLUOR," A\&A 475, 243-250 (2007).

[13] Absil, O., Di Folco, E., Mérand, A., Augereau, J., Coudé du Foresto, V., Defrère, D., Kervella, P., Aufdenberg, J. P., Desort, M., Ehrenreich, D., Lagrange, A., Montagnier, G., Olofsson, J., ten Brummelaar, T. A., McAlister, H. A., Sturmann, J., Sturmann, L., and Turner, N. H., "A near-infrared interferometric survey of debris disc stars. II. CHARA/FLUOR observations of six early-type dwarfs," A $6 A$ 487, 1041-1054 (Sept. 2008).

[14] Akeson, R. L., Ciardi, D. R., Millan-Gabet, R., Merand, A., Folco, E. D., Monnier, J. D., Beichman, C. A., Absil, O., Aufdenberg, J., McAlister, H., Brummelaar, T. t., Sturmann, J., Sturmann, L., and Turner, N., "Dust in the inner regions of debris disks around a stars," ApJ 691, 1896-1908 (Feb. 2009).

[15] Absil, O., Mennesson, B., Le Bouquin, J.-B., Di Folco, E., Kervella, P., and Augereau, J.-C., "An interferometric study of the Fomalhaut inner debris disk I. Near-infrared detection of hot dust with VLTI/VINCI," ApJ 704, 150-160 (Oct. 2009).

[16] Hinz, P. M., Angel, J. R. P., Hoffmann, W. F., McCarthy, D. W., McGuire, P. C., Cheselka, M., Hora, J. L., and Woolf, N. J., "First results of nulling interferometry with the Multiple-Mirror Telescope," in [Astronomical Interferometry], R. D. Reasenberg, ed., Proc. SPIE 3350, 439-447 (July 1998).

[17] Colavita, M. M., Serabyn, E., Millan-Gabet, R., Koresko, C. D., Akeson, R. L., Booth, A. J., Mennesson, B. P., Ragland, S. D., Appleby, E. C., Berkey, B. C., Cooper, A., Crawford, S. L., Creech-Eakman, M. J., Dahl, W., Felizardo, C., Garcia-Gathright, J. I., Gathright, J. T., Herstein, J. S., Hovland, E. E., Hrynevych, M. A., Ligon, E. R., Medeiros, D. W., Moore, J. D., Morrison, D., Paine, C. G., Palmer, D. L., Panteleeva, T., Smith, B., Swain, M. R., Smythe, R. F., Summers, K. R., Tsubota, K., Tyau, C., Vasisht, G., Wetherell, E., Wizinowich, P. L., and Woillez, J. M., "Keck Interferometer Nuller Data Reduction and On-Sky Performance," PASP 121, 1120-1138 (Oct. 2009).

[18] Liu, W. M., Hinz, P. M., Hoffmann, W. F., Brusa, G., Miller, D., and Kenworthy, M. A., "Observations of Main-Sequence Stars and Limits on Exozodical Dust with Nulling Interferometry," ApJ 693, 1500-1507 (Mar. 2009).

[19] Hinz, P. M., "Detection of Debris Disks and Wide Orbit Planets with the LBTI," in [Exoplanets And Disks: Their Formation And Diversity], T. Usuda, M. Tamura, \& M. Ishii, ed., API Conference Series 1158, 313-317 (Aug. 2009).

[20] Absil, O., Coudé Du Foresto, V., Barillot, M., and Swain, M. R., "Nulling interferometry: performance comparison between Antarctica and other ground-based sites," A $6 A$ 475, 1185-1194 (2007).

[21] Ollivier, M., Absil, O., Allard, F., Berger, J., Bordé, P., Cassaing, F., Chazelas, B., Chelli, A., Chesneau, O., Coudé du Foresto, V., Defrère, D., Duchon, P., Gabor, P., Gay, J., Herwats, E., Jacquinod, S., Kern, P., Kervella, P., Le Duigou, J., Léger, A., Lopez, B., Malbet, F., Mourard, D., Pelat, D., Perrin, G., Rabbia, Y., Rouan, D., Reiss, J., Rousset, G., Selsis, F., Stee, P., and Surdej, J., "PEGASE, an infrared interferometer to study stellar environments and low mass companions around nearby stars," Experimental Astronomy 23, 403-434 (Mar. 2009).

[22] Danchi, W. C., Barry, R. K., Lawson, P. R., Traub, W. A., and Unwin, S., "The Fourier-Kelvin Stellar Interferometer (FKSI): a review, progress report, and update," in [Optical and Infrared Interferometry], Proc. SPIE 7013 (July 2008).

[23] Defrère, D., Absil, O., Coudé du Foresto, V., Danchi, W. C., and den Hartog, R., "Nulling interferometry: performance comparison between space and ground-based sites for exozodiacal disc detection," $A \& A 490$, 435-445 (Oct. 2008).

[24] Lisse, C. M., Chen, C. H., Wyatt, M. C., Morlok, A., Song, I., Bryden, G., and Sheehan, P., "Abundant Circumstellar Silica Dust and SiO Gas Created by a Giant Hypervelocity Collision in the 12 Myr HD172555 System," ApJ 701, 2019-2032 (Aug. 2009). 
[25] Booth, M., Wyatt, M. C., Morbidelli, A., Moro-Martín, A., and Levison, H. F., "The history of the Solar system's debris disc: observable properties of the Kuiper belt," MNRAS 399, 385-398 (Oct. 2009).

[26] Krivov, A. V., Löhne, T., and Sremčević, M., "Dust distributions in debris disks: effects of gravity, radiation pressure and collisions," A\&̊A 455, 509-519 (Aug. 2006).

[27] Thébault, P. and Augereau, J., "Collisional processes and size distribution in spatially extended debris discs," A\&A 472, 169-185 (Sept. 2007). 